\title{
Detecting the Magnetic Cosmic Web through Deep Radio Polarization Imaging
}

\author{
Lauren Hunt $^{1 *}$, Russ Taylor ${ }^{1,2,3}$ Kurt van der Heyden ${ }^{1}$ \\ ${ }^{1}$ University of Cape Town \\ ${ }^{2}$ Inter-University Institute for Data Intensive Astronomy \\ ${ }^{3}$ University of the Western Cape \\ E-mail: lauren@ast.uct.ac.za
}

\begin{abstract}
Magnetic fields are present throughout the Universe and many astrophysical processes are influenced by their presence. One of the largely unknown aspects of cosmic magnetism is that of the cosmic web and how magnetic fields behave in large scale structure. Due to the indirect nature of the detection of these fields, it is difficult to determine much about the origin and evolution of magnetic fields. One method of detection is to measure how the polarisation angle of light is rotated in the presence of electrons and a magnetic field. In this paper, we simulate the Rotation Measure (RM) experienced by radio emission as it travels from an extragalactic source to the observer in large-scale simulations. Our aim is to investigate the effect of extragalactic magnetic fields on the spatial distribution of RM. This paper aims to produce RM maps of the simulated universe and use these to inform upcoming radio surveys and how we can use observations to investigate the cosmic web.
\end{abstract}

MeerKAT Science Workshop - MeerKAT2016-,

25-27 May, 2016,

Stellenbosch, South Africa

${ }^{*}$ Speaker. 


\section{Introduction}

An understanding of large-scale magnetic fields is essential to a full understanding of the evolution of the universe. On an extragalactic scale, magnetic fields have observed in galaxies and clusters. In galaxies, ordered and turbulent magnetic fields are observable on all scales, while interstellar fields can drive galaxy dynamics [1]. Magnetic fields in the intergalactic medium can be produced by jets from AGN, galactic winds and the motion of gases in the intergalactic medium. On the scale of the large-scale structure of the universe, magnetic fields may exist in filaments, similar to those seen in dark matter simulations [2].

The polarisation of radio emission is one of the most powerful probes of magnetic fields in the cosmos. Much of the radiation that we observe from astrophysical sources in the radio band is emitted as linearly polarised synchrotron radiation. Magnetized plasmas that lie in the propagation path of the radiation will cause a change in the polarisation angle through the mechanism of Faraday rotation.Cosmic Faraday Rotation is negligible at high-frequency, but becomes significant in the radio at $\mathrm{GHz}$ frequencies and below. By observing the frequency dependence of Faraday rotation we can determine the Rotation Measure (RM) and thereby make inferences about the magnetic fields through which the light ray passed [3].

We use large-scale universe simulations to investigate the effect of extragalactic magnetic fields on the spatial distribution of Rotation Measure (RM) of radio sources that will be detected in deep radio images with MeerKAT. We aim to inform MeerKAT International GigaHertz Tiered Extragalactic Exploration Survey (MIGHTEE) observations to detect magnetic fields associated with large-scale structure.

\section{Simulations}

The objective of this work is to simulate the path of light as it travels from a radio source to an observer. In order to achieve our aim, we make use of existing large-scale simulations to determine what environments the light ray passes through. This allows us to calculate the RM that results from environments like galaxies and the intergalactic medium in the path of light. We do this in order to construct RM maps which can be analysed to see if the structure is detectable at different configurations of the magnetic field parameters.

\subsection{Universe simulation}

We use the MUFASA simulations [4] to populate the universe. These are cosmological hydrodynamical simulations that make use of the meshless finite mass method in GIZMO [5]. This simulation contains snapshots of volume $50 h^{-1} \mathrm{Mpc}^{3}$ up to $z=4$. Each snapshot contains roughly $2.5 \times 10^{8}$ particles in the form of dark matter, gas and stars. Galaxies and halos in the simulations were identified using SPHGR [6], which is a data reduction suite that processes the outputs from the GADGET code. It returns the positions and attributes of galaxies and halos from the MUFASA snapshots.

The snapshots and SPHGR output are merged into a light cone with diameter of $1.2^{\circ}$ to a redshift of $z=1$ using a routine from M. Cawood [7]. The light cone, illustrated in (Figure 1), shows the matter distribution in the first snapshot of the simulation in blue and the first section of 


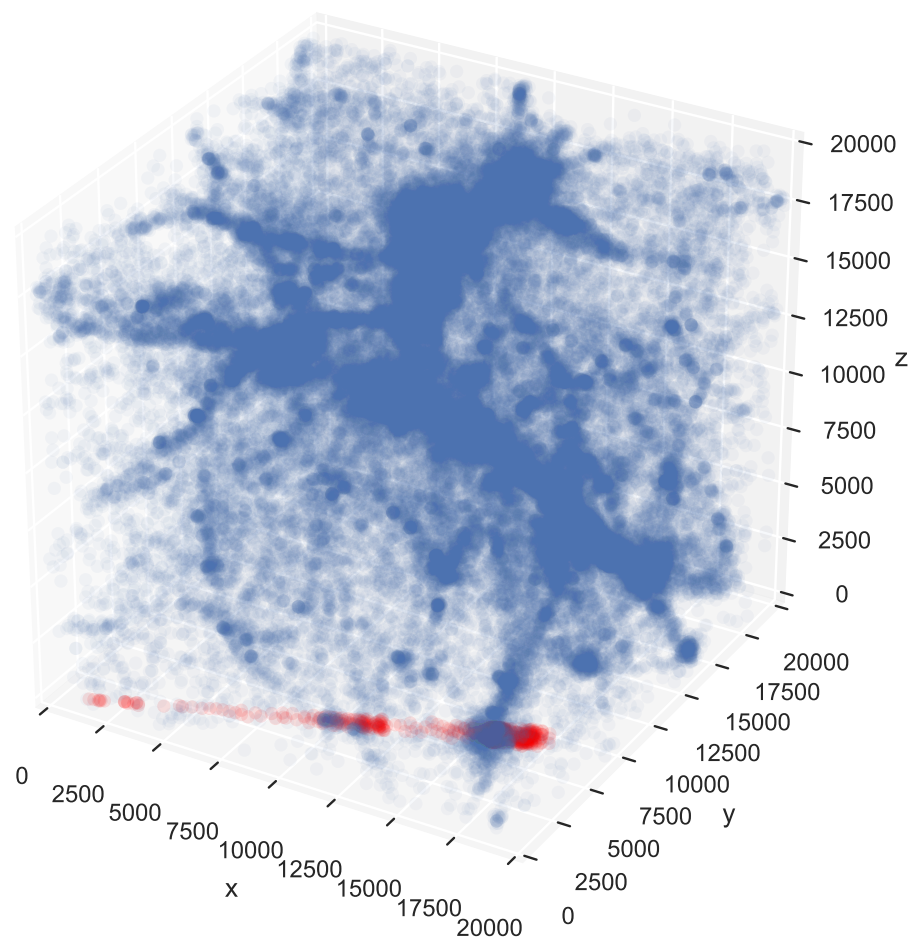

Figure 1: The red data points represent the small pencil beam that is extracted from the larger simulated data cube which is represented by the blue data points.

the cone is shown in red. The Faraday Rotation effect of magnetic fields that may be embedded in large-scale structure is simulated by tracing a line-of-sight through the volume.

\subsection{Radio sources and Ray Tracing}

Polarised radio sources are placed at the back of the light cone at random angular positions. In future work, more realistic radio source distributions will be created using the SPHGR galaxy catalogue. Each source is attributed an intrinsic rotation measure sampled from a normal distribution with $\mu=0$ and $\sigma=6 \mathrm{rad} \mathrm{m}^{-2}$ [8]. The path of the ray through the light-cone is divided into ray elements every $10 \mathrm{kpc}$, to estimate parameters on a scale that is comparable to the size of the smallest objects in the simulation.

To calculate the contribution of each element to the Rotation Measure, we must determine what objects the light ray passes through along the line-of-sight. This can be very computationally intensive. We use kdTrees to search for particles near to the light ray. This is a spatial partitioning technique that repeatedly cuts the data in half according to its distribution and stores the information in a tree to allow for easy searching. This allows us to execute an efficient search for gas, galaxies and halos that were close enough to a ray element to have an effect. This resulting information is used to calculate the contribution to the Rotation Measure in each segment. 


\subsection{Contribution from gas and astrophysical objects}

We calculate the Faraday effect of gas, galaxies and halos separately. For the gas particles, we average properties of the nearby particles to calculate the effect on that segment of the ray. We ensure that we sample only the gas that lies outside of clusters or galaxies and assign a field strength $|B|(\mathrm{nG})$ to the gas according to

$$
|B|=1 \times 10^{6}\left(B_{0} \times N_{e}\right),
$$

where $N_{e}$ is the electron density, $B_{0}$ is the strength of the magnetic field set at $10 \mathrm{nG}$ [9] and the scale factor $1 \times 10^{6}$ normalises the electron density so that our magnetic field distribution averages to $B_{0}$.

We account for material that flowed out from galaxies, and carrying magnetic fields with it, by assigning higher magnetic fields when metallicity is greater than 0 . according to

$$
\mathrm{B}=\mathrm{B}_{\mathrm{MHD}}\left(\frac{\rho}{\rho_{\text {scale }}}\right)^{\alpha}
$$

where $\rho$ is the density, $\rho_{\text {scale }}$ is a dimensionless scaling factor that is set to 4.0 here and $\mathrm{B}_{\mathrm{MHD}}$ is the magnetic field in the gas (see Equation 2.1). The exponent $\alpha=\frac{2}{3}$ indicates the dependence of the outflow fields on the gas density [10].

We do not have any clusters in this simulation and so we do not need to consider the magnetic fields associated with these structures. We consider only the central galaxies of each halo assuming the others are satellites. If the central galaxy is star-forming, then we use the magnetic field of that galaxy for the magnetic field of the object. We no not apply magnetic fields to halos under the assumption that their fields are symmetrical and therefore any Faraday Rotation would sum to 0 [11].

\section{Results}

The galaxy and halo fields are still in progress, but the magnetic field contribution from the gas has been taken into account in the ray tracing. We have used magnetic fields where the magnetic field is aligned along the direction of the light ray as well as randomly aligned. We also define a 'realistic' field where the field direction is in the direction of highest density in a $5 \mathrm{Mpc}$ radius with the assumption that if our current position has non-zero density, then the direction of highest density should point along a filament. Where the density along the ray is zero, we obtain a magnetic field strength of 0 and therefore assign no direction. Figure 2 shows the sum of the source RM and large scale structure RM with the realistic field direction for each source.

We use the second-order structure function, defined by

$$
S(\Delta \theta)=<[\operatorname{RM}(\theta)-\operatorname{RM}(\theta+\Delta \theta)]^{2}>
$$

to measure angular structure in the distribution of RM. Here $<>$ indicates an average value for all $\mathrm{RM}$ measurements in a given $\Delta \theta$ bin.

The structure functions that we obtained for the sources, random magnetic fields,realistic direction fields and for the case of a uniform large-scale field in the direction of the observer are shown in Figure 3. There is a higher structure throughout the function for the aligned field. As the methods are improved, we expect more structure to be evident. 


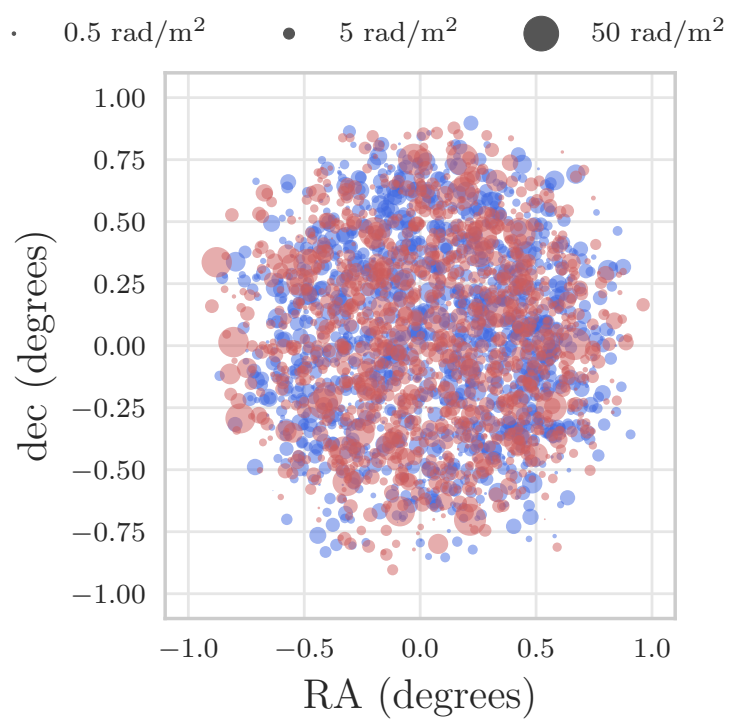

Figure 2: Rotation measure map of the rotation measure observed after the rays have passed through the LSS. The blue circles indicate a positive rotation measure and red circles indicate a negative rotation measure. The size of the circles indicate the magnitude of the RM.

\section{Summary}

We have begun a study to use universe simulations to assess the ability of deep radio imaging projects over scales of several square degrees to detect and measure the properties of magnetic fields associated with extragalactic environments and large-scale structure. Up to this point, we have been focused on creating the software to apply fields to the simulations and extract Rotation Measures. We will explore the parameter space of our models to assess the effect of IGM fields on the structure function. This paper aims to produce RM maps that incorporate many of the astrophysical processes that generate magnetic fields. Simulating the rotation measures that result in light travelling through the large scale structure will lead to a better understanding of what can be expected from RM maps from the MIGHTEE project.

\section{References}

[1] R. Beck, Cosmic magnetic fields: Observations and prospects, in AIP Conf. Proc., vol. 1381, pp. 117-136, 2011. arXiv: 1104.3749v1. DOI.

[2] T. Akahori, K. Kumazaki, K. Takahashi and D. Ryu, Exploring the intergalactic magnetic field by means of Faraday tomography, Publ. Astron. Soc. Japan 66 (jun, 2014) 65, [1403. 0325].

[3] R. Fitzpatrick, Faraday Rotation, in Maxwell's Equations Princ. Electromagn., ch. Electromag. 2008.

[4] R. Davé, R. Thompson, P. F. Hopkins, A. O., B. I. K., B. A. E. et al., mufasa: galaxy formation simulations with meshless hydrodynamics, Mon. Not. R. Astron. Soc. 462 (nov, 2016) 3265-3284.

[5] P. F. Hopkins, A new class of accurate, mesh-free hydrodynamic simulation methods, Mon. Not. R. Astron. Soc. 450 (apr, 2015) 53-110, [1409.7395]. 


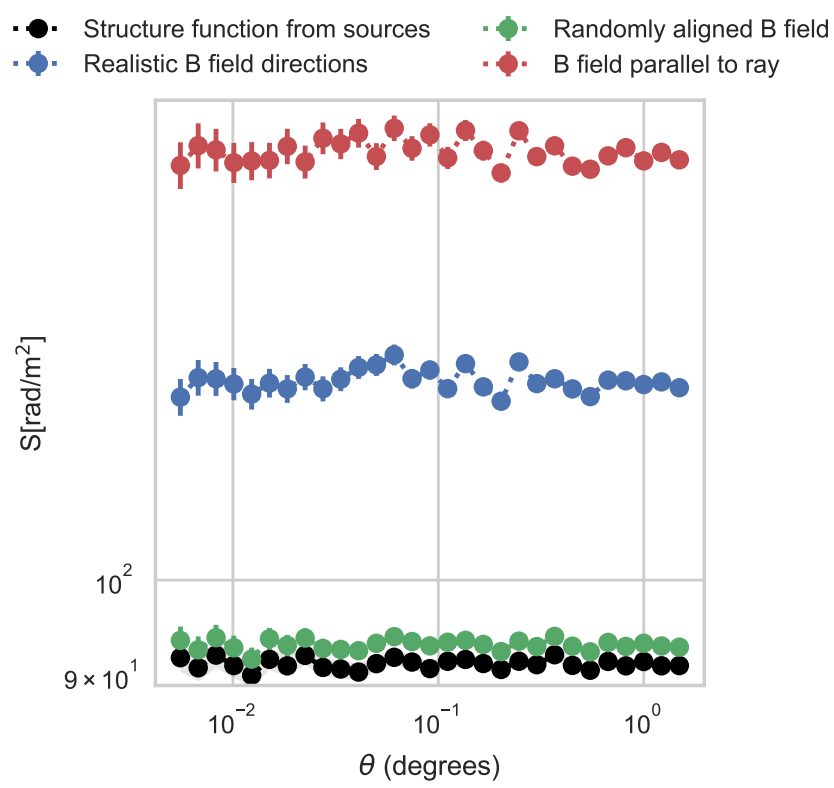

Figure 3: Figure comparing the structure functions for different directional choices for the magnetic fields. The errors on each bin are the the error on the mean for each bin

[6] R. Thompson, SPHGR: Smoothed-Particle Hydrodynamics Galaxy Reduction, Astrophys. Source Code Libr. 1 (2015) 2012.

[7] M. R. Cawood and M. Roy, HI Lightcones for LADUMA using Gadget-3 : performance profiling and application of an HPC code, .

[8] D. H. F. M. Schnitzeler, The latitude dependence of the rotation measures of NVSS sources, 1011.0737.

[9] T. Akahori and D. Ryu, Faraday Rotation Measure due to the Intergalactic Magnetic Field, eprint arXiv 1009 (nov, 2010) 570, [1107.0142].

[10] F. Stasyszyn, S. E. Nuza, K. Dolag, R. Beck and J. Donnert, Measuring cosmic magnetic fields by rotation measure-galaxy cross-correlations in cosmological simulations, Mon. Not. R. Astron. Soc. 408 (oct, 2010) 684-694, [1003. 5085].

[11] M. Soida, M. Krause, R.-J. Dettmar and M. Urbanik, The large scale magnetic field structure of the spiral galaxy NGC 5775, 1105.5259. 